\title{
Surgical Management of Oropharyngeal Squamous Cell Carcinoma
}

\author{
Kathryn M. Van Abel · Eric J. Moore
}

Published online: 8 June 2013

(c) Springer Science+Business Media New York 2013

\begin{abstract}
Squamous cell carcinoma of the oropharynx (OPSCC) occurs with an age-adjusted incidence rate of 2.2/ 100,000 per year, and makes up one of the largest growing subsets of head and neck cancer. Traditional risk factors include alcohol and tobacco exposure, however, the demographics have changed significantly over the past 2-3 decades, with human papillomavirus (HPV) infection now considered one of the most important risk factors. As our understanding of HPV-related OPSCC has developed, there has been increasing evidence that this new tumor subtype has a better overall prognosis and response to therapies. This review focuses on OPSCC from a surgical perspective. We review the relevant anatomy, diagnosis and workup, and discuss several different surgical approaches for management of this disease, including transoral robotic surgery, lateral oropharyngectomy and tonsillectomy, base of tongue resection, and neck dissection. In addition, the common complications associated with these treatment options are discussed.
\end{abstract}

Keywords Oropharyngeal cancer - Robotic surgery · Transoral surgery

Electronic supplementary material The online version of this article (doi:10.1007/s40136-013-0021-x) contains supplementary material, which is available to authorized users.

K. M. Van Abel · E. J. Moore ( $\square)$

Department of Otolaryngology, Head and Neck Surgery,

Mayo Clinic, 200 1st Street SW, Rochester, MN 55905, USA

e-mail: moore.eric@mayo.edu

K. M. Van Abel

e-mail: vanabel.kathryn@mayo.edu

\section{Introduction}

Head and neck cancer is the sixth most common cancer worldwide, with $12 \%$ of cases occurring within the oropharynx [1, 2]. Of those, up to $90 \%$ are diagnosed as squamous cell carcinoma (SCC). Less-common tumors in this location include the salivary gland, myoepithelial, soft tissue, hematolymphoid, and secondary tumors in addition to mucosal melanoma [3]. It is estimated that 5,000 new cases of oropharyngeal squamous cell carcinoma (OPSCC) are diagnosed each year in the US [4], with an annual age-adjusted incidence rate of 2.2/100,000 [5]. Risk factors for OPSCC have primarily long been chronic tobacco and alcohol exposure. However, even as national smoking and drinking trends have been decreasing, the incidence of OPSCC has surprisingly been increasing [1]. Extensive investigation has identified the human papillomavirus (HPV) as the likely cause of this epidemiologic shift and has resulted in extensive investigation into the etiology, evaluation, management and prognosis of this specific subtype of the disease.

Anatomy, Neurovasculature, Lymphatics

The oropharynx lies between the nasopharynx and hypopharynx/larynx, just posterior to the oral cavity. It is adjacent to critical neurovascular structures which must be considered intraoperatively (Fig. 1) [2]. The oropharynx is divided into four distinct subsites, including the tonsil, base of tongue (BOT), soft palate and posterior pharyngeal wall (PPW) $[2,6]$.

\section{Palatine Tonsils}

The palatine tonsils are located on the lateral oropharyngeal wall, bounded by the anterior and posterior tonsillar pillars, 
Fig. 1 Surgical anatomy of the oropharynx. The surgical anatomy of the oropharynx is complex and can be distorted by tumor growth. Understanding the relationship between these structures is critical to successful and safe surgical technique

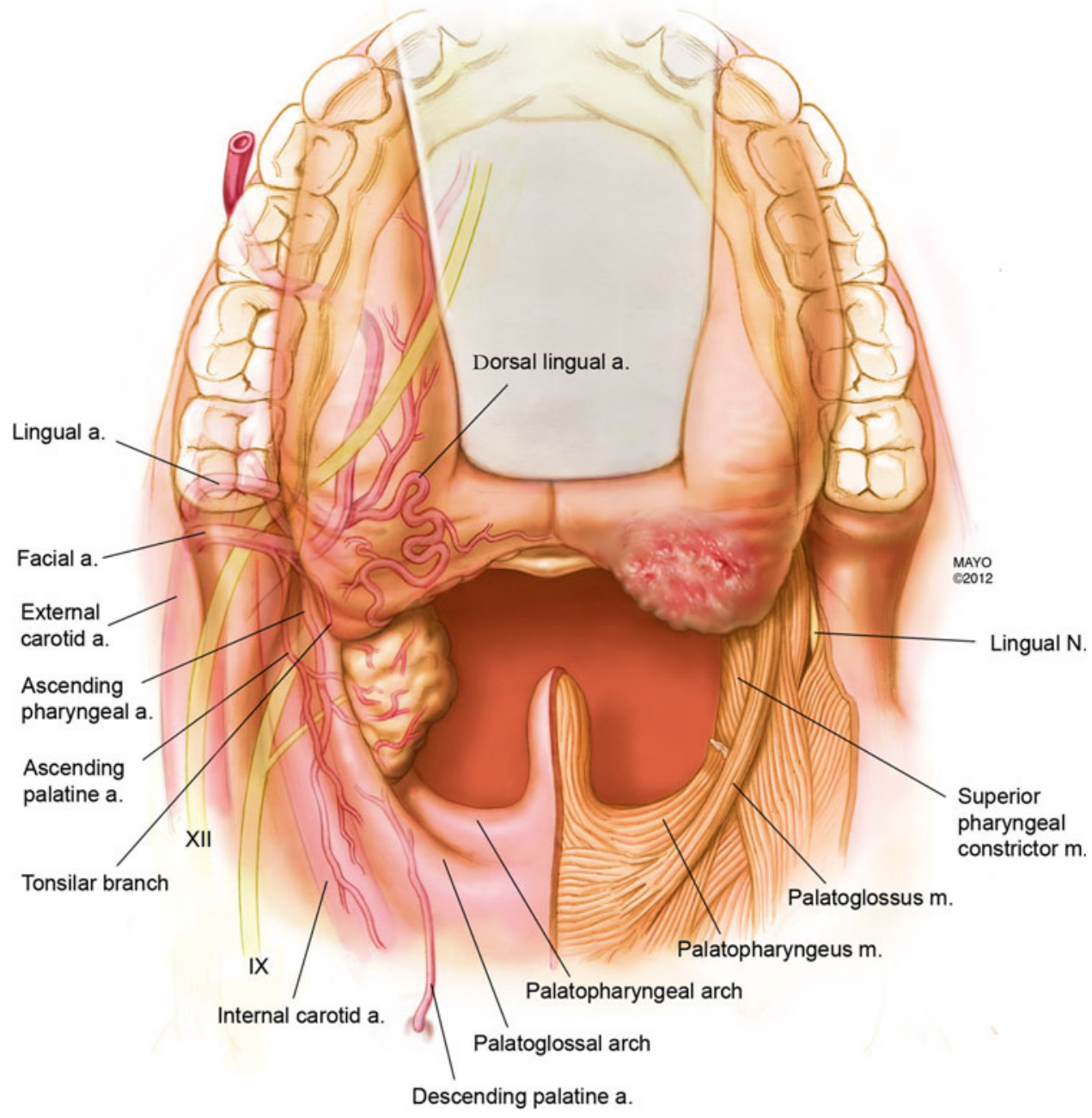

associated with the palatoglossus and palatopharyngeus muscle, respectively [7•]. Inferiorly, the palatine tonsil is separated from the BOT by the glossotonsillar sulcus, and superiorly it is limited by the confluence of the soft palate and tonsillar pillars. The palatine tonsil lies between these pillars, and is comprised of lymphoid tissue covered medially by stratified squamous epithelium, and laterally by a dense fascial capsule [7•]. The arterial supply of the tonsil is supplied by the external carotid system via both the tonsillar branch of the ascending pharyngeal artery and the descending palatine artery. The primary arterial supply, however, is the tonsillar branch of the facial artery [7•]. The tonsillar capsule is penetrated by a network of subepithelial lymphatics, which drain to the upper jugular nodes, with a predilection for the jugulodigastric nodes [6]. Metastatic spread to involve levels II, III, and IV are common [4], with spread to involve level V also possible [6]. The retropharyngeal lymph nodes are also at risk for spread from tonsillar SCC, most notably when tumors invade superiorly into the soft palate $[7 \bullet]$.

\section{Base of Tongue}

The BOT is the space found posterior to the circumvallate papilla, medial to the glossotonsillar sulci bilaterally, and superior to the level of the hyoid bone or the floor of the vallecula. The BOT includes the pharyngoepiglottic folds and the glossoepiglottic folds bilaterally. Just deep to the lingual tonsillar tissue lie the intrinsic tongue musculature superficially and extrinsic tongue musculature deep within the tongue [7•]. The arterial supply arises primarily from the external carotid system via the lingual artery. A dense system of lymphatics drain the BOT through channels that run along the lingual veins as they course through the pharyngeal wall to drain into the upper jugular nodes [8]. Each side of the BOT is connected via precollecting vessels which cross the midline to drain to the contralateral side $[6,8]$. According to Lindberg, levels II, III and IV were most commonly involved in BOT tumors, with bilateral metastases to level II being common [6]. 


\section{Soft Palate}

The soft palate is positioned between the palatopharyngeal and superior pharyngeal constrictor muscles, and is bordered anteriorly by the hard palate and posteriorly by the palatopharyngeus muscle and uvula.

The arterial supply arises from the internal maxillary system as the descending palatine artery, which branches into the greater and lesser palatine arteries [2]. The lesser palatine artery is the primary blood supply [9]. The lymphatics drain medially to the middle third of the jugular chain, laterally to the retropharyngeal lymphatics, and anteriorly to the hard palate and subsequently into the submental and submandibular nodal groups [2].

\section{Posterior Pharyngeal Wall}

The PPW spans from the level of the superior surface of the hyoid bone (or floor of the vallecula) inferiorly to the level of the soft palate superiorly. Its lateral limits are marked by the posterior pharyngeal pillars and the lateral extent of the piriform sinuses [2]. The arterial supply arises primarily from the ascending pharyngeal artery. The lymphatics drain primarily into the retropharyngeal lymph nodes, which then preferentially drain to level II and III $[6,8]$. In addition, there is also direct drainage from the PPW to levels II and III [8].

\section{Etiology and Epidemiology}

Typically, OPSCC presents in the 6th and 7th decade in patients with a history of heavy tobacco and alcohol use [10]. Therefore, as the rate of cigarette smoking in the United States and Europe decreased, it was naturally predicted that a similar decrease in the incidence of OPSCC would occur. However, while the incidence of tobacco related, HPV-negative, HNSCC has decreased as expected, the overall incidence of OPSCC in the US and Europe has risen rather dramatically $[11,12 \bullet \cdot]$. During the past two decades, this increase has been most notable in men in the US, who present in their 40s to 50s, with either no history or a less significant history of exposure to tobacco or alcohol [10-14]. Numerous studies have now documented the role of the HPV in up to $25 \%$ of head and neck SCC patients. Up to $60-70 \%$ of OPSCC, particularly in the tonsil and base of tongue, has been associated with HPV $[11,13]$. In fact, HPV infection has now surpassed tobacco as the major risk factor for OPSCC $[1,11,14]$.

\section{Clinical Presentation and Initial Evaluation}

In a study by Pitchers et al. [15], the most common presenting symptoms for OPSCC were a neck mass (49.3\%), sore throat $(33.3 \%)$, lesion seen by the patient $(5.9 \%)$, incidental finding by a physician $(2.9 \%)$, referred otalgia $(2.9 \%)$, dysphagia $(2.9 \%)$ and globus pharyngeus $(1.4 \%)$. When taking a history, it is also important to ask about hemoptysis, weight loss, trismus, limited tongue mobility and odynophagia [16].

\section{Diagnosis}

The National Comprehensive Cancer Network (NCCN) recommends a thorough history and physical exam, tissue diagnosis, HPV testing for prognosis (suggested), chest imaging, CT scan with contrast and/or MRI of both the suspected primary site and the neck, 18-fluorodeoxyglucose positron emission tomography and CT (PET/CT) scan for stage III-IV disease (suggested), dental evaluation as indicated, and examination under anesthesia if necessary [17].

Staging OPSCC depends on the TNM system described by the AJCC [18]. The "T stage" is determined by the size of the primary tumor and invasion into local structures. T1-T3 tumors are described based on size alone, and do not demonstrate invasion into adjacent structures. T4a OPSCC demonstrate invasion into the larynx, deep/ extrinsic tongue musculature, medial pterygoid muscles, hard palate, or mandible [18]. The features that differentiate $\mathrm{T} 4 \mathrm{~b}$ tumors include invasion into the lateral pterygoid musculature, pterygoid plates, lateral nasopharynx, skull base, or encasement of the carotid artery [18]. T stage is therefore determined based on a combination of physical exam, imaging, and pathological factors.

Due to the inability to detect micrometastases, Rodrigo et al. [19] note that CT, MRI, US, and PET/CT have a sensitivity of only $80-85 \%$ for detecting nodal disease [20]. Therefore, the gold standard for determining the "N stage" is based on pathologic evaluation of nodal tissue [21]. Distant metastasis at presentation are rare (2-5\%) $[22,23]$, and most commonly involve the lung, liver and bone [24]. PET/CT scanning has offered a novel technique for evaluating for the presence of distant disease [25•].

\section{Prognosis}

Prognosis for OPSCC depends largely on the stage and site of disease [26]. Currently, there is no conclusive evidence that surgical versus nonsurgical treatment results in a significant difference in survival for OPSCC. The recognition that HPV positive tumors have a better prognosis has led some to believe that these represent a distinct disease process from the more traditional HPV negative tumor subtypes [27, 28]. 


\section{Management}

Patients with exophytic, mobile, T1-3/N0-N1 tonsil and BOT cancers (with anatomical features which allow transoral exposure) may be candidates for transoral surgery alone [29]. Patients with T4 primary tumors and/or N2-N3 disease are candidates for transoral surgery plus adjuvant therapy.

The surgeon should think of transoral surgery as one approach to the oropharynx. It is less important whether the surgeon chooses transoral robotic surgery or transoral laser microsurgery: these are both transoral approaches, which differ in instrumentation only. The goal of all transoral oropharyngeal cancer resection is complete tumor removal with pathologically negative margins, complete removal of involved cervical lymphatics, secondary intention healing with remucosalization, and rapid return to function. If the surgeon cannot assure the patient a high probability of this scenario, then alternative treatment should be considered.

The contraindications for transoral surgery can be divided into oncologic, anatomic, and physiologic issues. The oncologic contraindications are fixation of the tumor, direct extension to the cervical lymph nodes, extension of tumor to the lateral pharyngeal wall, distant metastasis/ unresectable nodal disease/skin metastasis, or involvement of $>50 \%$ of the BOT or to such an extent that major flap reconstruction would be necessary to close defect (relative contraindication). The anatomic contraindications include severe trismus, pharyngeal/tongue crowding preventing adequate tumor exposure, and the presence of a retropharyngeal carotid artery [30, 31]. Physiologic contraindications are related to patient comorbidities that would prevent general anesthesia or decrease the ability to heal by secondary intention.

\section{Surgical Approaches}

\section{Transoral Robotic Surgery}

Operative treatment begins with general anesthesia with a laser-safe endotracheal tube and paralysis for the duration of the transoral portion of the procedure. Preoperative antibiotic prophylaxis with coverage for oropharyngeal organisms should be given. Oropharyngeal tumors can complicate anesthesia induction and airway exposure, and the surgeon should communicate clearly with the anesthesiologist to develop a safe airway plan. After intubation or tracheotomy, the surgeon should carefully palpate the oropharynx to evaluate the submucosal extent and mobility of the tumor. Fixation to the lateral pharynx or skull base is a contraindication to proceeding with a transoral approach.

The teeth should be protected using molded thermoplastic sheeting (WFR/Aquaplast Corp, Wyckoff, New
Jersey) prior to exposing the tumor with an oral retractor. The Feyh-Kastenbauer (FK) laryngeal retractor (Gyrus ACMI, Southborough, Massachusetts) is the most frequently used; although other oral retractors such as the Crowe Davis can be useful. It is suspended from the left side of the surgical bed with a Storz laryngoscope holder (Karl Storz, Tuttlingen, Germany). The da Vinci S Surgical System is then brought to the left side of the patient and the $0^{\circ}$ binocular endoscope is positioned to allow an optimal view. The EndoWrist 5-mm Schertel grasper (Intuitive Surgical, Inc) is attached to the left robotic arm, the 5-mm spatula cautery is attached to the right robotic arm, and the arms are advanced into the field. The surgeon sits at the remote console and manipulates the instruments for tumor removal while an assistant sits at the head of the bed to assist with suctioning the field and providing hemostasis.

\section{Lateral Oropharyngectomy and Tonsillectomy (Video 1)}

The technique for transoral surgical removal of tonsil tumors has been described by Weinstein et al. [32] and Holsinger et al. [33]. The mucosa is incised at the retromolar trigone, and dissection is carried to the pterygomandibular raphe. This landmark guides the surgeon to a dissection plane between the pharyngeal constrictor muscle, which becomes the lateral margin of tumor resection, and the buccopharyngeal fascia. The palatal cuts are made, taking care to avoid the superior extent of the tumor. Normally, the medial palatal cut extends just medial to the uvula, but the uvula can be transected if the tumor extends to the midline of the palate. The dissection is continued laterally and inferiorly to the level of the styloglossus and stylopharyngeus muscles. The glossopharyngeal nerve may be visualized anterolaterally to these muscles. Numerous vascular branches of the lingual, facial, and ascending pharyngeal arteries and veins course between the fascia and superior constrictor muscles. Bleeding can be controlled by additional cautery, or branches of the lingual artery or tonsillar artery can be managed with vascular clips. If the lingual or tonsillar arteries are encountered, at least two clips should be applied on the patient side with a Storz laryngeal clip applier (Karl Storz).

Care should be taken to keep the buccopharyngeal fascia intact, if possible, to protect the thin layer of fat in the parapharynx around the carotid artery and to prevent communication into the neck. If the extent of a tumor into this area is questionable on preoperative examination or imaging, we perform ipsilateral neck dissection and place a cotton patty medial to the carotid artery before beginning the transoral procedure. In this manner, we isolate and protect the vasculature from instruments during the transoral dissection. Additionally, the lingual and facial arteries can be ligated to assist with vascular control. 
The mucosa is cut at the soft palate medial to the tumor, and dissection is carried through the constrictor muscle on the PPW to the level of the prevertebral fascia. The medial superior pharyngeal constrictor muscle is dissected bluntly from the prevertebral fascia, the styloglossus and stylopharyngeus muscles are transected inferiorly, and the dissection is carried into the lingual lymphoid tissue until the inferior extent of the tumor is safely mobilized and the tumor is freed with a margin of normal tissue. The assistant grasps the tumor, maintaining orientation, and the tumor is removed from the mouth and prepared for pathology.

\section{Transoral Base of Tongue Resection (Video 2)}

Prior to transoral BOT resection, the surgeon should carefully palpate the base of tongue to gain an appreciation for the extent of the tumor. Once the retractor is placed, the confines of the oropharynx can be distorted. The surgeon should place the retractor that offers the widest exposure of the tumor. This will differ based on tumor location, size, and patient anatomy.

After exposure is maximized, the surgeon makes a vertical cut through the tumor to determine its greatest depth. This is helpful because horizontal (tangential to tumor) cuts across the BOT that fail to take into account the full tumor depth may inadvertently leave tumor in the deep intrinsic tongue muscle. After determination of the depth of the tumor, a vertical medial cut is made through the intrinsic tongue muscle to the hyoid bone, a horizontal cut is made deep to the tumor to allow it to drop into the pharynx for better visualization, and finally a lateral cut is made through the tonsillar fossa, pharyngeal mucosa, and pharyngoepiglottic fold, if necessary. The surgeon should be aware laterally of the dorsal lingual artery and contributions of the lingual artery and generously apply clips to attain hemostasis. If the tumor extends deeply and laterally beyond the hyoid bone to the hyoglossus muscle, it is important to appreciate the proximity of the lingual artery and hypoglossal nerve in this region. In these situations, an external approach to the tumor may be safer and allow for more confident resection margins.

\section{Margins, Hemostasis and Reconstruction}

Frozen-section analysis of the tumor and precise communication between the surgeon and the pathologist are critical to the success of complete transoral tumor removal. Tissue in any area that might have residual tumor, as suggested by physical examination, visual appearance, or pathology findings, should be excised and examined histologically. Often, additional tissue from the margins is taken as indicated until frozen-section histology confirms that circumferential microscopic tumor removal is complete.
The retractor is released during the frozen section margin analysis to prevent unnecessary venous congestion and resultant edema of the tongue. After the margins have been cleared, the retractor is reopened, the defect is inspected, and hemostasis is ensured. Flaps have been utilized in TORS of the tonsil, but in our experience, unless the lateral pharyngeal wall is significantly resected, the wound will contract and heal by secondary intention and the patient's speech and swallowing will benefit from the contracture and mucosalized surface.

\section{Open Approaches}

With growing enthusiasm for transoral approaches, the surgeon should not abandon open approaches, which are still useful in selected OPSCC. Bulky and deep tumors of the BOT can be resected with a combination of transoral and transhyoid pharyngotomy exposure, allowing management of the hypoglossal nerve and lingual artery, providing excellent appreciation of tumor boundaries, while still avoiding the morbidity of mandibulotomy. In surgical resection of T4 lateral pharyngeal tumors and in salvage surgery of bulky or deep BOT tumors, the surgeon should still consider the use of mandibulotomy and potential flap reconstruction.

\section{Neck Dissection}

After complete tumor removal, the retractor is removed and the patient is prepped and draped for unilateral or bilateral neck dissection. Patients typically undergo ipsilateral selective neck dissection (II-IV) for N0-N2b and N3 OPSCC. The retropharyngeal nodes are removed if patients have obvious metastasis on imaging or if tumors substantially invade the soft palate or PPW. Although some surgeons stage the neck dissection to prevent a communication between the oropharynx and neck, we have not found this to be necessary. The advantages of simultaneous neck dissection are multiple, including access to the vessels supplying the oropharynx during the operation, cost and time savings [34]. After neck dissection, suction drains are placed and the mouth is flooded with saline to rigorously investigate the parapharynx for oral communication. Communications are closed by suturing the constrictor muscle either transorally or through the neck, closing a portion of the digastric muscle to the sternocleidomastoid muscle lateral to the defect, and placing Tisseel (Baxter Bioscience, Deerfield, Illinois) over the defect on the neck side. The neck incisions are meticulously closed, and the surgeon evaluates the airway endoscopically to decide whether extubation is safe and hemostastis is adequate. It is very important to evaluate for hemostasis at the end of the case prior to extubation, as an unrecognized bleeding vessel may result in aspiration upon extubation. A nasogastric feeding 
tube is placed if there is a communication between the oropharynx and the neck or if the extent of the defect may compromise swallowing. Patients undergoing TORS for tonsil cancer rarely require an alternate airway, but if there is concern, a tracheostomy can be placed, or extubation can be postponed until airway edema resolves.

\section{Post-operative Care}

We routinely admit our TORS patients to the general care floor. The immediate post-operative course is focused on transitioning to oral intake of a pureed/soft diet and medications, removing neck drains, and pain management. Oropharyngectomy is a painful procedure, therefore, narcotic analgesia is administered IV and then transitioned to PO. If the patient has inadequate $\mathrm{PO}$ intake secondary to pain, then medications and nutrition are administered via NGT until PO intake improves. Patients are generally discharged from the hospital after 2-3 days. Patients are advised to avoid lifting $>10 \mathrm{lbs}$, refrain from exertional activities, avoid medications and supplements that effect blood coagulation, and avoid driving for 2 weeks. Typically, pain and PO intake improve substantially at 2 weeks post-operation.

The patient is seen back at 10-14 days after discharge and instructed on diet, swallowing therapy, physical therapy of the neck and shoulders, and activity level. If they were discharged with an NGT, we obtain a swallow study and evaluation with a speech pathologist. If they demonstrated adequate swallow function and airway protection, the NGT is removed.

\section{Adjuvant Therapy}

Individual patient and tumor differences make protocol care and general recommendations impossible. In general, patients who use tobacco heavily or currently with node positive tumors (HPV+ or HPV-) with adverse pathologic factors are offered postoperative adjuvant chemoradiotherapy (concurrent cisplatinum and 6000 rads IMRT), nonsmoking or distant former smokers with HPV positive tumors with $\mathrm{N} 2 \mathrm{~b}$ and above disease are offered adjuvant radiotherapy (6000 rads IMRT), and HPV+N0-N2a patients are offered surgery alone. Our results with this treatment have demonstrated locoregional and distant control that are as good or superior to any other large published series of tonsil cancer with acceptable cost and excellent treatment related morbidity $[35,36]$.

\section{Complications}

\section{Bleeding}

Bleeding after oropharyngeal resection can be minor or major. Minor mucosal bleeding can occur anytime in the first 2 weeks after the operation, but is more common at 9-14 days and presents as blood-tinged saliva that can last for minutes to hours. This bleeding can usually be managed with conservative measures including ice water, digital pressure, avoidance of anticoagulants, and observation. Major bleeding presents with copious arterial bleeding from named vessels in the tonsillar fossa (facial artery), BOT (lingual/dorsal lingual artery), or supraglottis (superior laryngeal artery). This can occur due to erosion into vessels that were initially controlled during the operation or vasodilation of vessels that constricted and retracted during the operation. This bleeding rarely results in hypotension or exsanguination, but it can result in airway compromise and death [37]. The surgeon can minimize this possibility with meticulous intraoperative hemostasis, careful dissection, clip application to major vessels, and ligation of the tonsillar and lingual artery during neck dissection; however, the possibility cannot be completely eliminated. The patient needs to be counseled on this possibility, and all members of the team must be well versed in management of oropharyngeal bleeding and airway protection [37].

\section{Neck Abscess}

Neck abscesses can result from unrecognized communication between the oropharynx and neck. This usually presents as delayed swelling and erythema in the neck, and it will result in spontaneous drainage and wound breakdown if not recognized and managed. Prevention hinges on meticulous intraoperative closure of any communication as described above. If a neck abscess occurs despite these measures, it should be recognized early and managed by opening the incision in a dependant area, draining, culturing the abscess, cleaning the cavity with saline and peroxide, packing daily with iodoform gauze until the wound is closed, and initiating appropriate antibiotics.

\section{Tongue/Dental Paresthesia}

Paresthesias can result from retractor pressure in prolonged cases, patients with large tongues, or narrow mandibular arches. Management is conservative as symptoms usually resolve in several days.

\section{Velopharyngeal Insufficiency and Rhinolalia}

Tumors with superior extension that necessitate palatal resection can result in palatal insufficiency. If $<50 \%$ of the palate is removed, theses symptoms will improve over the subsequent 3-4 months. The defect should scar and contract as the contralateral palate fills the space laterally and posteriorly, thus narrowing the velopharyngeal inlet. For tumors that require resection of $>50 \%$ of the palate, 
a pharyngeal flap, buccal fat or mucosa pedicled flap may be necessary to obturate the palate.

\section{Delayed Pharyngeal Healing}

Delayed pharyngeal healing can occur in patients treated with prior- or postoperative radiotherapy, malnutrition, immunodeficiency, or persistent tumor. Delayed healing will present with pain and ulceration at the surgical site. If this persists for more than 3 months postoperatively, biopsy is indicated to rule out persistent tumor. In the absence of tumor, optimizing the wound environment using hyperbaric oxygen, antibiotics, and nutritional support can speed remucosalization.

\section{Conclusion}

As our understanding of OPSCC has been rapidly changing over the past 20-30 years, so too has our management. Surgeons must remain flexible as we investigate the response of OPSCC to surgical and non-surgical management in an effort to maximize both functional and oncologic outcomes. For select OPSCC tumors, transoral resection with neck dissection alone can offer excellent outcomes, and for more extensive nodal disease, the combination of transoral resection with adjuvant therapy can result in de-escalation of radiation and potential avoidance of chemotherapy. The surgeon must also remain facile in open approaches for when transoral resection is contraindicated, and must understand the important role for adjuvant therapy. Finally, regardless of approach used, success is determined by careful consideration of individual tumor characteristics and adherence to the principles of oncologic management.

Disclosure Kathryn M. Van Abel and Eric J. Moore declare that they have no conflict of interest.

Compliance with Ethics Guidelines This article does not contain any studies with human or animal subjects performed by any of the authors.

\section{References}

Papers of particular interest, published recently, have been highlighted as:

- Of importance

•- Of major importance

1. D'Souza G, Kreimer AR, Viscidi R, et al. Case-control study of human papillomavirus and oropharyngeal cancer. N Engl J Med. 2007;356(19):1944-56. doi:10.1056/NEJMoa065497.

2. Harreus U. Cummings otolaryngology: head \& neck surgery. 5th ed. 5(null) ed. Mosby, Inc.
3. Barnes L, Eveson JW, Reichart P. WHO Blue Books. Lyon: IARC; 2005.

4. Lin DT, Cohen SM, Coppit GL, Burkey BB. Squamous cell carcinoma of the oropharynx and hypopharynx. Otolaryngol Clin N Am. 2005;38(1):59-74.

5. Surveillance, Epidemiology, and End Results (SEER) Program (www.seer.cancer.gov) SEER*Stat Database: Populations-Total U.S. (1969-2009)<Single Ages to 85+, Katrina/Rita Adjustment $>-$ Linked To County Attributes-Total U.S., 1969-2009 Counties, National Cancer Institute, DCCPS, Surveillance Research Program, Surveillance Systems Branch, released April 2011.

6. Lindberg R. Distribution of cervical lymph node metastases from squamous cell carcinoma of the upper respiratory and digestive tracts. Cancer. 1972;29(6):1446-9.

7. - Moore EJ, Janus J, Kasperbauer J. Transoral robotic surgery of the oropharynx: clinical and anatomic considerations. In: Richards A, editor. Clin Anat. 2011;25(1):135-141. doi:10.1002/ca.22008. This article provides an excellent review of the anatomy and surgical technique used for TORS OSPCC resection.

8. Werner JA, Dünne AA, Myers JN. Functional anatomy of the lymphatic drainage system of the upper aerodigestive tract and its role in metastasis of squamous cell carcinoma. Head Neck. 2003;25(4):322-32. doi:10.1002/hed.10257.

9. Hollinshead HW. Hollinshead. Philadelphia: Harper and Row; 1954. p. 1-280.

10. Toner M, O'Regan EM. Head and neck squamous cell carcinoma in the young: a spectrum or a distinct group? Part 1. Head Neck Pathol. 2009;3(3):246-8. doi:10.1007/s12105-009-0135-0.

11. Marur S, D’Souza G, Westra WH, Forastiere AA. HPV-associated head and neck cancer: a virus-related cancer epidemic. Lancet Oncol. 2010;11(8):781-9.

12. • D'Souza G, Dempsey A. The role of HPV in head and neck cancer and review of the HPV vaccine. Prev Med. 2011;53 Suppl 1:S5-S11. doi:10.1016/j.ypmed.2011.08.001. This article offers important insight into the role of HPV in head and neck cancer, with a special emphasis on future vaccine applications.

13. Sedaghat AR, Zhang Z, Begum S, et al. Prognostic significance of human papillomavirus in oropharyngeal squamous cell carcinomas. Laryngoscope. 2009;119(8):1542-9. doi:10.1002/lary.20533.

14. Näsman A, Attner P, Hammarstedt L, et al. Incidence of human papillomavirus (HPV) positive tonsillar carcinoma in Stockholm, Sweden: an epidemic of viral-induced carcinoma? Int J Cancer. 2009;125(2):362-6. doi:10.1002/ijc.24339.

15. Pitchers M, Martin C. Delay in referral of oropharyngeal squamous cell carcinoma to secondary care correlates with a more advanced stage at presentation, and is associated with poorer survival. Br J Cancer. 2006;94(7):955-8. doi:10.1038/sj.bjc.6603044.

16. Cohan DM, Popat S, Kaplan SE, Rigual N, Loree T, Hicks WL Jr. Oropharyngeal cancer: current understanding and management. Curr Opin Otolaryngol Head Neck Surg. 2009;17(2):88-94. doi: 10.1097/MOO.0b013e32832984c0.

17. Pfister DG, Ang KK, Brizel DM, et al. Head and neck cancers. J Natl Compr Cancer Netw. 2011;9(6):596-650.

18. Deschler DG, Day T. TNM staging of head and neck cancer and neck dissection classification: American academy of Head and Neck. 2008:1-88.

19. Rodrigo JP, Shah JP, Silver CE, et al. Management of the clinically negative neck in early-stage head and neck cancers after transoral resection. Head Neck. 2011;33(8):1210-9.

20. U S, M D, C W, et al. Is there a need for positron emission tomography imaging to stage the N0 neck in T1-T2 squamous cell carcinoma of the oral cavity or oropharynx? Ann Otol Rhinol Laryngol. 2008;117(11):854-863.

21. Van Abel KM, Moore EJ. Focus issue: neck dissection for oropharyngeal squamous cell carcinoma. ISRN Surg. 2012;2012: 547017. doi:10.5402/2012/547017. 
22. Ruzevick J, Olivi A, Westra WH. Metastatic squamous cell carcinoma to the brain: an unrecognized pattern of distant spread in patients with HPV-related head and neck cancer. J Neurooncol. 2013. doi:10.1007/s11060-013-1075-9.

23. Bailey BJ, Johnson JT, Newlands SD. Head \& Neck Surgery: Otolaryngology. Philadelphia: Lippincott Williams \& Wilkins; 2006.

24. Chung TS, Stefani S. Distant metatases of carcinoma of tonsillar region: a study of 475 patients. J Surg Oncol. 1980;14(1):5-9. doi:10.1002/jso.2930140103.

25. - Spector ME, Chinn SB, Rosko AJ, et al. Diagnostic modalities for distant metastasis in head and neck squamous cell carcinoma: are we changing life expectancy? Laryngoscope. 2012;122(7): 1507-1511. doi:10.1002/lary.23264. This study offers an important look at the use of technology for ongoing oncologic surveillance and questions current practice changes regarding the use of PET/ CT scan.

26. Mendenhall WM, Morris CG, Amdur RJ, et al. Definitive radiotherapy for tonsillar squamous cell carcinoma. Am J Clin Oncol. 2006;29(3):290-7. doi:10.1097/01.coc.0000209510.193 $60 . \mathrm{f} 9$.

27. Gillison ML, D'Souza G, Westra W, et al. Distinct risk factor profiles for human papillomavirus type 16-positive and human papillomavirus type 16-negative head and neck cancers. J Natl Cancer Inst. 2008;100(6):407-20. doi:10.1093/jnci/djn025.

28. Gillison ML, Chaturvedi AK, Lowy DR. HPV prophylactic vaccines and the potential prevention of noncervical cancers in both men and women. Cancer. 2008;113(10 Suppl):3036-46. doi: $10.1002 /$ cncr.23764.

29. Grant DG, et al. Oropharyngeal cancer: a case for single modality treatment with transoral laser microsurgery. Arch Otolaryngol Head Neck Surg. 2009;135(12):1225-30.
30. Moore EJ, Olsen KD, Kasperbauer JL. Transoral robotic surgery for oropharyngeal squamous cell carcinoma: a prospective study of feasibility and functional outcomes. Laryngoscope. 2009;119(11): 2156-64. doi:10.1002/lary.20647.

31. Van Abel KM, Carlson ML, Moore EJ. Symptomatic internal carotid artery medialization: a rare anatomic variant resulting in cough, dysphonia, and dysphagia. Clin Anat. 2013. doi:10.1002/ ca.22178.

32. Weinstein GS, et al. Transoral robotic surgery: radical tonsillectomy. Arch Otolaryngol Head Neck Surg. 2007;133(12): 1220-6.

33. Holsinger FC, McWhorter AJ, Menard M, Garcia D, Laccourreye O. Transoral lateral oropharyngectomy for squamous cell carcinoma of the tonsillar region: i. technique, complications, and functional results. Arch Otolaryngol Head Neck Surg. 2005;131(7):583-91. doi:10.1001/archotol.131.7.583.

34. Moore EJ, Olsen KD, Martin EJ. Concurrent neck dissection and transoral robotic surgery. Laryngoscope. 2011;121(3):541-4. doi: 10.1002/lary.21435.

35. Hinni ML, Moore EJ. Cost considerations in the treatment of oropharyngeal squamous cell carcinoma. Otolaryngol Head Neck Surg. 2012;146(6):946-51.

36. Moore EJ, Olsen SM, Laborde RR, et al. Long-term functional and oncologic results of transoral robotic surgery for oropharyngeal squamous cell carcinoma. Mayo Clin Proc. 2012;87(3): 219-25. doi:10.1016/j.mayocp.2011.10.007.

37. Salassa J, Hinni M, Grant D, Hayden R. Postoperative bleeding in transoral laser microsurgery for upper aerodigestive tract tumors. Otolaryngol Head Neck Surg. 2008;139(3):453-9. doi:10.1016/ j.otohns.2008.06.010. 